\title{
AN APPROACH TO SELF-SHIELDING SYSTEMS OF RADIOTHERAPY
}

\author{
RANA JAMAL ABD ELRAHIM HOURANI \\ Imam Abdul Rahman Bin Faisal University, Dammam, KSA
}

\begin{abstract}
The overall economic benefit of improving access to radiotherapy resources provides a great incentive to invest in areas that are severely short of treatment services. These investments could save 26.9 million years of age for patients treated in these areas. In radiotherapy, there is an urgent need to address the burden of cancer that is increasingly facing most countries in the world. Although current innovations focus on more specialized devices and treatments, a new approach to radiotherapy is being proposed through self-shielding systems for the cancer burden. There are devices that are a 6 MV linear self-acceleration system that uses diagnostic-quality rays scan instructions. Over $90 \%$ of the cancer markers can be treated, the cost of this system is estimated at less than \$1 million, and only requires minimal shielding of rooms. The device's diversity and low cost makes it an ideal system to address global inequalities in resources of radiotherapy. The results indicate that all necessary self-shielding for the audience and neighboring individuals is integrated as part of the system design in order to remove the heavy workload, which eliminates the need to build a dedicated radiation depot.
\end{abstract}

KEYWORDS: Shielding Systems, Cancer Treatments \& Radiotherapy

Received: Mar 04, 2020; Accepted: Mar 24, 2020; Published: Jul 13, 2020; Paper Id.: IJMPERDJUN2020309

\section{INTRODUCTION}

Radiotherapy is a tool that produces significant benefits by controlling and treating human suffering. Half of cancer patients are estimated to benefit from radiotherapy to treat disease, mitigate or control local. Although radiotherapy can lead to many benefits, it is often not underutilized, even in high-income countries that have the ability to treat patients effectively. In order to reach the goal set by 2030, efforts must be made in the area of prevention and other factors, but this alone will not solve the problem. Therefore, it is clear that a comprehensive approach to increasing access to radiotherapy facilities and supportive resources must be at the forefront of this issue (Atun, 2015).

Radiation therapy is treatment using high energy X-rays, as high levels of radiation work to kill cancer cells and prevent them from growing and dividing. Radiation therapy is used to treat cancer because cancer cells grow and divide more quickly than most other normal cells around it. Nature cells also seem to have a greater ability to fully recover from the effects of radiation from cancer cells. Radiotherapy can be used alone, or in combination with chemotherapy and surgery, or both. Radiation therapy does not cause any pain or discomfort or discomfort, as it is more like taking an ordinary X-ray, but it requires the child to remain still for several minutes more. In some cases, children should be given some sedatives to stay still throughout the radiotherapy period (Benedict, 2014).

During the past years, the demand for radiation sources and devices has increased in establishing radiation therapy programs to treat cancer patients with the assistance of the International Atomic Energy Agency. This assistance is usually provided through technical cooperation projects. Providing this equipment and assistance without a systematic approach to radiation shielding systems in clinical aspects, dose dosage, safety, and 
maintenance can cause and endanger patient treatment outcomes (either with unacceptable frequency or double rates), and may lead to an unacceptable risk of accidents (INTERNATIONAL ATOMIC ENERGY AGENCY VIENNA, 2008). Therefore, it must be recognized that both an overdose and an underdosage are undesirable, which leads to a possible increase in the frequency of tumors and possible complications of treatment. In addition, all implemented projects must comply with international basic safety standards for the safety of radiation sources and for protection from ionizing radiation (FOOD AND AGRICULTURE ORGANIZATION OF THE UNITED NATIONS,, 1996).

\section{PROBLEM STATEMENT}

Many innovations in radiation therapy are constantly taking place. From the early days of relatively unguided radiotherapy, the field of radiotherapy developed to new levels with many improvements in accuracy, uniformity, and image and dose orientation. Whether tremendous improvements come from the BrainLab Vero device with stepped linear accelerator and multi-image guidance systems, or on the other hand a linear accelerator guided by MRI in ViewRay to photograph soft tissues in real time, it seems that radiotherapy focuses on highly specialized treatments that increase productivity The accuracy of the facility. Although these advanced systems constantly create new ways of treatment that were not available before, they offer these advanced and innovative systems a cost that can exceed millions of dollars even though they constantly create new methods of treatment that were not available before, and also require employees Highly trained. Although developments in these systems are deservingly popular and serve a useful purpose for many patients who need radiotherapy, there is room for radiotherapy that requires attention worldwide, there are many middle and low income countries that only have medicine little radiation therapy, which makes treatment matters more complicated, and as the population ages and grows, this disparity in radiotherapy systems and resources continues to widen ( International Atomic Energy Agency., 2017). To address this problem and the complex burden, there must be a global effort and innovation coordinated with a new focus to provide high-quality, versatile, low-cost radiation therapy to meet treatment for all levels. Therefore, radiotherapy self-shielding systems can be used to address this problem.

Although these systems can be the most expensive, it will be able to treat most cancer indicators. Applying these systems to increase radiotherapy resources worldwide can reduce the overall economic burden that this problem represents. Moreover, unlike the common narrative, current studies provide solid evidence that expanding global access to radiation therapy may provide important economic return in most areas of significant under-service provision. Therefore the research problem lies in this main question: "what are the Self-shielding Systems of radiotherapy and what the requirements for designing these systems are?"

\section{RESEARCH SIGNIFICANCE}

The research draws its importance from the importance of the subject we will address, which is the topic of "Self-shielding Systems of radiotherapy", which represent a worldwide issue and an important area of research. Therefore, conducting such a research regarding this topic is expected to have a high positive reflections and significance that can be summarized as in the following:

- The importance of the research lies in its use primarily by health physicists, medical physicists and other radiation protection professionals in planning and designing new armor systems for new radiotherapy.

- It provides guidance on self-shielding systems for radiotherapy and describes how the required radiation shielding should be determined. 
- This study would represent a good reference for the future studies as long as it would provide the subsequent researchers and interested scholars in the field of Health physicists and medical physicists with valued literature, recommendations and suggestions that are important for their proposed studies in the light of contemporary.

\section{LITERATURE REVIEW}

\section{Radiation Fields and Sources}

The interaction of ionizing radiation with the material and its transport through the material is essential for the analysis and design of shielding systems in radiotherapy. Analysis and design are two sides of the same coin, as they work together. First: In design, the permissible radiation dose, source intensity, or dose rate at a specific location is determined, and the primary task of design is by creating the necessary protection and determining its type. Second: In the analysis, the main shielding material is determined, and the dose is determined, by the severity of the source, or by looking at the first dose. Radiation is depicted as a particle - neutrons, electrons, photons, and so on. The term Ioinzing radiation refers to the transmission of energy in the form of electromagnetic waves (electromagnetic) or atomic-secondary molecules so that the energy is sufficient to cause the ionization of matter. Ionizing radiation is a type of energy released by specific atoms in the form of waves or electromagnetic particles (Van Dye, 2013).

The field of ionizing radiation includes radioactive materials, resonators (x-rays), and catalysts used by medical institutions, industrial laboratories, and research institutes. The term radiation field also refers to particles and their paths in some open areas or across some closed areas, per unit of time or during a specific time period (Khan, 2003).

Characterization of the radiation field for any one type of radiation particle requires determination of the spatial variation of the particle's direction and energy distribution. Certain rotational properties may be required for complete characterization in some cases, such as those encountered in neutron dispersion experiments. The following sections describe how to characterize the radiation field in a space region in terms of the impact of particles and how to characterize the radiation field at the boundaries in terms of particle flow. Flow and radiation are called radiative quantities as distinct from dose quantities. The concepts of flow and flow apply to both measurement and calculation. The measured quantities are inherently random, as they involve enumerating the tracks of individual particles. Measurement requires boundary areas or limited quantities. It also applies to calculated flow or fluency by Monte Carlo methods, because these calculations are mostly computer simulations of experimental decisions. The flow or flow is treated as an imperative point function and should be interpreted as the expected value, in the statistical sense, of a random variable. It is perfectly appropriate to refer to the amount of flow, flow, or the amount of dose associated with a point in space. However, it must be recognized that any measurement is only one estimate of the expected value (Shultis \& Faw, 2000).

There are tens of millions of new cases of cancer each year worldwide, according to estimates by the International Agency for Research on Cancer (IARC), with just over half of the cases in developing countries (Coleman, Norman, \& Minsky., 2015).

The distribution of cancer cases among the sexes around the world is somewhat equal. As cancer rates increase with increasing age, the majority of new cases occur in the age group of over 65 years. However, age distributions of cancer vary greatly between developed and developing countries; there are many more cases of cancer in childhood, adolescence and youth in developing countries, while cancer of the elderly dominates in developed countries (Stewart BW, 2015).

In general, the most common types of tumors in the world are lung cancer, followed by breast cancer, colorectal 
cancer, stomach cancer, and prostate cancer. In males the most common type of cancer is lung cancer, followed by the prostate and stomach. In females, breast cancer is the most common type of tumor, followed by cervical cancer. The incidence of different types of cancer varies with different regions ( International Atomic Energy Agency., 2017).

\section{Proposed System: SS-HQIGRT}

This new device is called a Self Shielded radiotherapy system, or SS-HQIGRT, this device is designed to be a fairly low cost option that can be used for all income levels in any facility or hospital. This proposed system also uses diagnostic quality computed tomography technology, which offers several benefits over alternative X-ray methods, see figure 1.

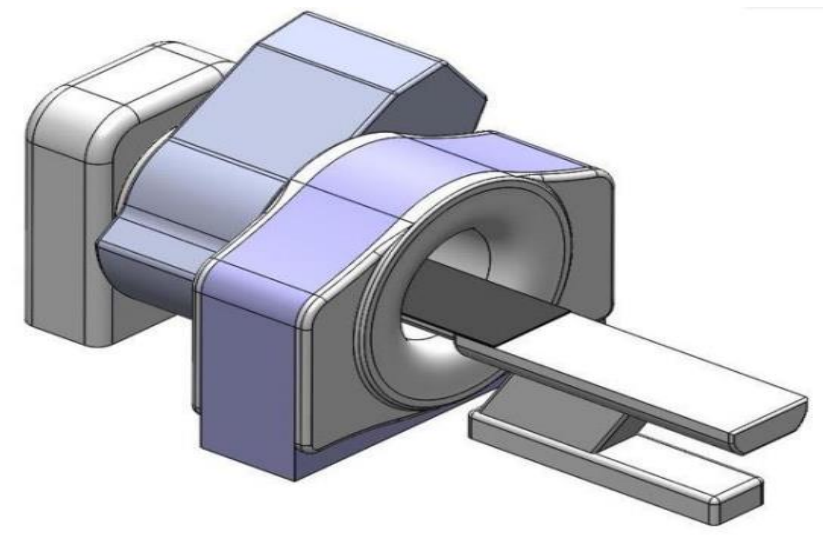

Figure 1: The proposed design for the SS-HQIGRT system (Lechuga, 2017).

\section{Self-Shielding Design}

This proposed system uses negative methods to reduce all shielding requirements and radiation levels. For this, a typical cellar is designed, with the primary shielding belt intercepting the expected initial radiation field, by creating a diluted barrier that wraps around the ceiling and walls. The SS-HQIGRT system transports this basic belt close to the patient, thus reducing the protected area significantly.

Compared to conventional linac systems with a $1 \mathrm{~m}$ distance from source and axis, the proposed SS-HQIGRT system would shorten that distance to $0.85 \mathrm{~m}$. Thus, increase the patient's dose rate, and thus to reduce the time needed for radiotherapy and thus reduce the use factor. Due to the proposed system field size, the initial shielding width will be reduced compared to the typical linear field size.

Moreover, the SS-HQIGRT system is restricted to move along a circular path other than systems like CyberKnife and others. This restriction will greatly reduce the space needed for the base shield. These features will reduce the required protection significantly and clearly, but there will still be a large amount of protection required in such circumstances. 


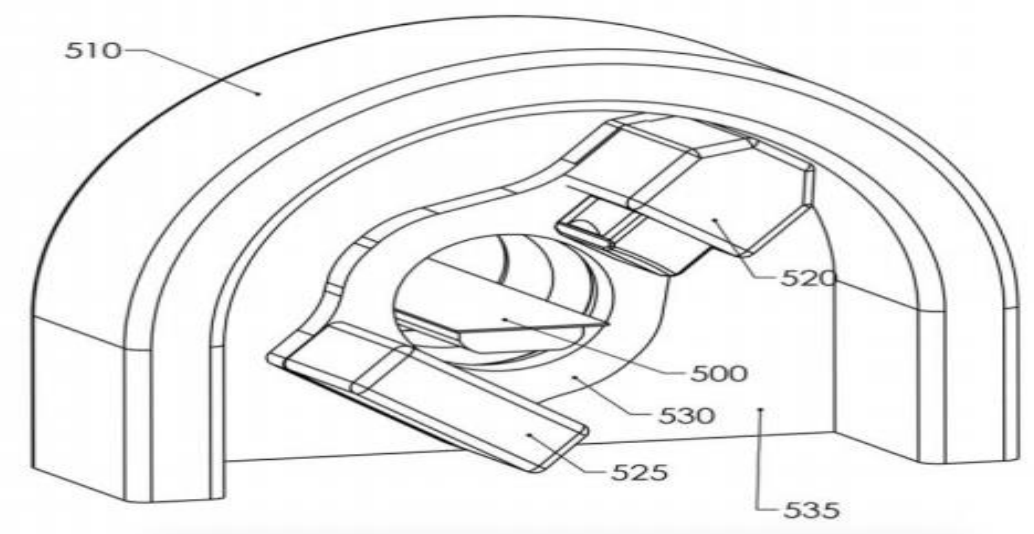

Figure 2: Proposed embodiment of SS-HQIGRT shows the primary shielding and beam stop (Lechuga, 2017).

To deal with the initial radiation, the SS-HQIGRT system will reduce the radiation from the treatment beam with a solid lead bracket, which will intercept the treatment beam at every gantry angle, as shown in Figure 2. In addition, the lead beam stopper will be installed at the bottom of the isocenter center corresponding to the treatment head in order to further reduce the initial radiation. The arc mounted on the ground combines with the beam stopper to form a 5-lead TVL value for the $6 \mathrm{MV}$ photon energies. TVL is a layer of diluent that reduces the intensity of incoming radiation to one-tenth of the value received. According to NCRP Report 151 (2005), the lead TV length of the TV is about $5.7 \mathrm{~cm}$ for $6 \mathrm{MV}$, so the base armor thickness will be $28.5 \mathrm{~cm}$. The arc will be fixed to the ground and will maintain a constant distance from the center of the corners across all corners. Scattered radiation is mainly mitigated by the lead arc, but there is some scattered radiation that will not be intercepted by the base shield. Scattered radiation that is not intercepted will be absorbed by a five cubic centimeter steel shield that can be attached to the facility walls. Moreover, there will be radiation emitted by the parallel head SSHQIGRT. Leakage radiation will be mitigated by the lead shield that is installed around the system processing head. However, some radiation that is not absorbed by the treatment head guard will be absorbed by the lead arch (Lechuga, 2017).

\section{Benefits and Applications}

The SS-HQIGRT system is characterized by many advantages and wide applications that are possible in the field of radiotherapy, as follows (Lechuga, 2017):

- As the first thing about this system is that it compares well with current radiotherapy systems by offering similar capabilities and benefits, but at a minimal cost. Because it can be used as a main inpatient treatment regimen for the ability to treat more than $90 \%$ of cancer markers

- This system can equip poor and rural areas with a cost-effective radiotherapy system, for rural areas with very low population densities may benefit from mobile radiotherapy site. Because of the minimum required secondary protection, the proposed system can be modified to become mobile.

- SS-HQIGRT can be fully implemented without basement within four weeks vs. 1-1.5 years for conventional systems.

- This system can enable poor or rural areas that currently do not have options for radiotherapy to invest in a lowcost system that will have the ability to treat the vast majority of existing cancer indicators.

- This will allow the radiotherapy system to move to rural areas of lack of services and for which no radiotherapy 
clinic may be located nearby.

- The proposed system can be used as an overflow system either to absorb increased patient load, or to relieve stress on higher systems. Since the proposed system has the potential to implement within weeks and can treat most indicators of cancer, it can act as a backup system whenever a large influx of patients causes immersion in the clinic.

- SS-HQIGRT system can be used as the primary treatment system; many clinics can have multiple advanced systems which is a preferred system due to the added advantages and patient productivity.

- It may be useful to convert some of the patient's pregnancy to SS-HQIGRT in order to maintain advanced systems for indicators requiring high precision and additional features since many indicators of cancer can be cured without having advanced features.

Usually, radiotherapy systems are used inside the clinic, where patients are sent from the primary care facility to the clinic for treatment. This process may be difficult for patients who have been hospitalized for other reasons (Elekta., 2017). Transportation to and from these clinics may require an ambulance with a variety of medical equipment required. This process may be costly and difficult for the patient, but the suggested solution via SS-HQIGRT is to install an on-site treatment room in the primary care facility, to treat patients who may have difficulty moving off-site. This solution will provide an option for patients in the absence of movement to receive the care needed with a relatively small investment for the primary care facility. Due to the self-protection nature of SS-HQIGRT, the primary care facility can use almost any room of sufficient size, and the ability to support additional steel protection as a treatment room. Moreover, this system can act as a quick and cost-effective alternative to older radiotherapy devices. Devices like Co-60 systems can create security risks due to highly active radioactive sources, and fail to provide treatment according to current standards. At the lowest cost in the current industry, SS-HQIGRT provides state-of-the-art CT scan and computerized tomography and meets the imperfect needs of the current radiotherapy market. This system addresses the need for a low-cost system that does not require expensive basement, can be installed relatively quickly, and provides treatment for most cancers, and represents those regions and communities that need radiotherapy ability (Lechuga, 2017).

There are many different forms of radiation therapy, as many different forms are replaced and renewed on the market (Lechuga, 2017). Many linacs are outdated as newer, faster and more accurate platforms are offered. Some of the Cobalt-60 systems are replaced by modern linac systems that can produce higher energy photons without radioactive waste, due to their short half-life, as they require periodic maintenance (Benedict \& et al., 2014). There are many systems available and developed on the market today, from companies such as Varian Medical Systems, Elekta, ViewRay, Brainlab and Accuray, a wide range of treatments are available from very versatile and very wide, to very specific and exotic types. These systems can provide radiation by gamma rays, protons, or x-rays. However, more robust and sophisticated systems tend to significantly increase the cost of the system used, the level of personnel specialization, and the protection requirements for the vault to be installed in the facility. The purpose of this research is to discuss the systems in the market, and to study their limitations and their capabilities (Elekta., 2017).

A new radiotherapy system developed by View Ray (Cleveland, Ohio) is being introduced in many areas. View Ray MRIdian includes a combination of MRI and radiotherapy to provide increased soft tissue contrast and continuous imaging during treatment. The MRIdian received a FDA license to treat patients in 2012. Furthermore, since then, the University of California at Los Angeles, University of Washington and others have installed the MRIdian system (ViewRay., 
2017). View Ray is completely innovative because it relies on non-ionizing radiation to visualize the patient for planning, positioning and treatment. View Ray literature assesses that MRIdian has special benefits over linac's traditional radiotherapy systems. The claim is that MRI provides increased contrast in the soft tissue areas. Furthermore, this discrepancy can result in clearly defined margins that can safely classify affected tissues. Moreover, MRI can be used continuously during treatment to track and adapt to tumor movement. This continuous imaging has the added benefit of not providing an additional dose to most patients (ViewRay., 2015).

Zap-X system is a new compact and self-contained radiosurgery system built and developed in San Carlos, California by ZAP Surgical Systems, Inc. This new device is concerned with the treatment of stereotactic radiosurgery (SRS) for malignant and benign cervical spine lesions (in some cases such as C7). The linear accelerator is the $2.7 \mathrm{MV}$ bandwidth radiation source (linac). linac is installed in close proximity to the large gyroscope within a group of moving columns, provided with radiation protection, as each of these columns revolves around a common center between them. This linac beam design enables the crossover of the M1 Citrine equivalent at a solid angle, as ideally required in the SRS of cranial (Weidlich G A., 2017).

Therapeutic beam placement is accomplished with great precision by precise movements of the patient's automatic schedule, and two independent accelerator cycles. Most of the components needed to produce the package, such as electronics that cause the beams, the waveguide system, and the radio frequency power source, are installed or combined with radiation shielding in the field of the patient's rotary treatment room (Benedict \& et al., 2014).

Zap-X achieves accurate patient $3 \mathrm{D}$ registration with an integrated $\mathrm{kV}$ flat imaging system $(\mathrm{kV})$ and this system also rotates around the patient's head. Pairs of non-axial X-ray images and image-to-image linkage are used to determine the original location of the patient's anatomy with respect to the center of the device, before and during radiotherapy (Elekta., 2017). The patient is heavily supported at the treatment table that extends outside the treatment field and is surrounded by intense radiation protection during radiosurgery. The table guard consists of a pneumatic door and a swivel frame on a steel frame (NCRP, 2014). 

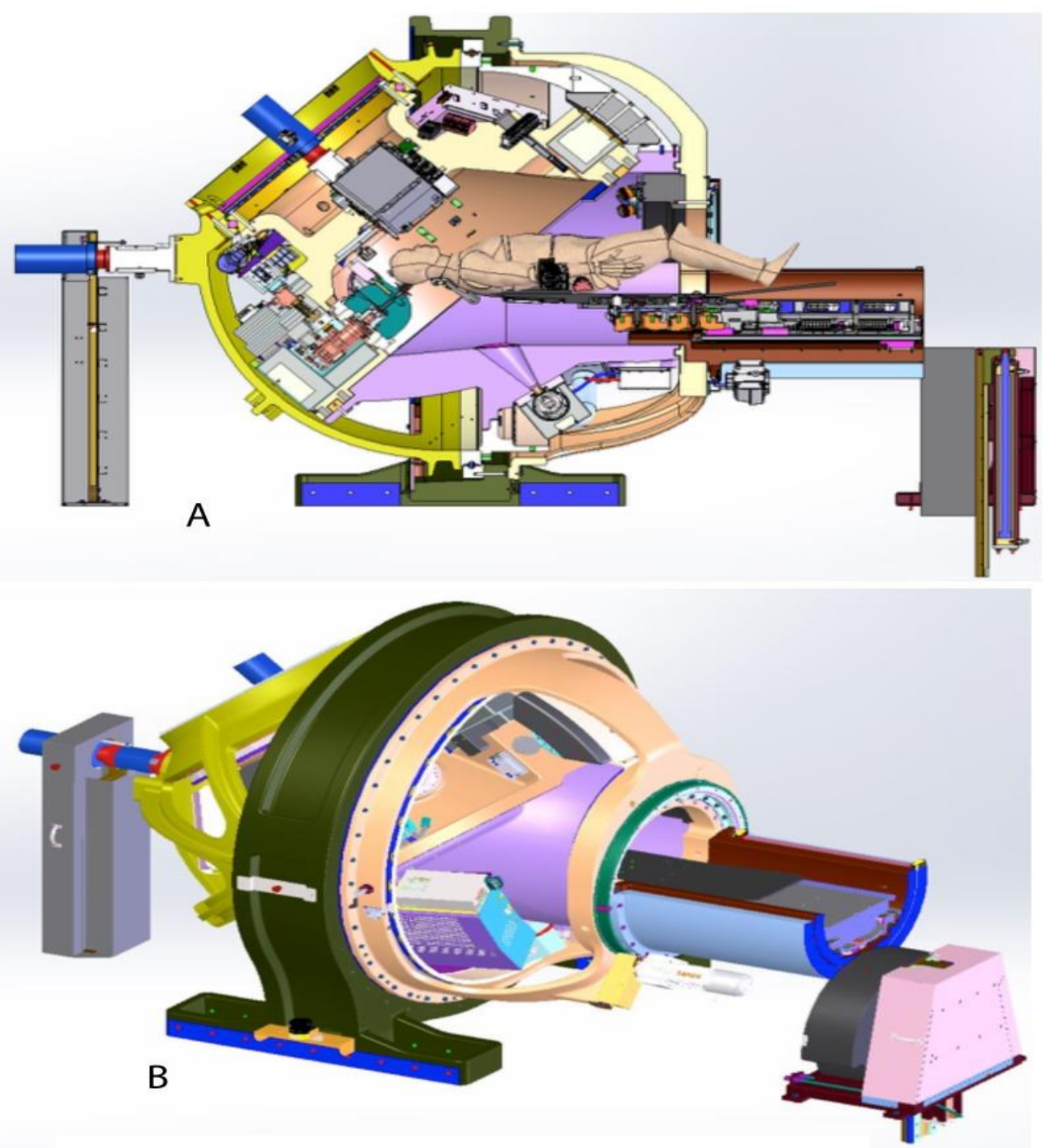

Figure 3: The Zap-X system and its shielding ((A) Cross-sectional view of the Zap-X system; (B) Room's eye view of the Zap-X system.) (Weidlich G A., 2017).

\section{MATERIALS AND METHODS}

Initially, the treatment table shell is designed for patients, and the shielded chamber, in addition Along with rotating beam stop, by Monte Carlo simulation, in order to provide the greatest effect of self-shielding. The shielding effect will be provided in traditional systems through the ceiling, floor and walls of the radiotherapy cellar. Where the objectives of this system are as follows (Weidlich G A., 2017):

- Provide full shielding for workers outside the safety zone of one meter in length from the perimeter of the Zap-X 
system to levels acceptable to the public. This limit generally applies to radiation workers and non-radiation workers mentioned by the National Radiation Protection Council (NCRP, 2014).

- Provide shielding at any point along the ocean line, which results in instantaneous exposure rate of no more than 2.0 milligrams in any one hour.

- Meet the design requirements of being self-shielded.

The shielding consists mainly of iron; however, as appropriate, there are also high $\mathrm{Z}$ supplementary materials consisting of tungsten, lead or steel (iron) alloys. In terms of design, the thickness and materials to be used, as well as the location of radiation shielding mode, are determined using the radiation transfer mathematical simulation based on the Monte Carlo BEAMnrc dose algorithm (Rogers DW, 1995 ). The beam is designed based on the engineering of beam-determining components such as the target of primary and secondary stabilizers and $\mathrm{x}$-rays. The validity of the beam pattern is validated based on a comparison with the measured attenuation properties of the shielding materials used (Siebers JV, 2005). Consideration is taken of radiation leakage and scattering, in addition to the initial radiation, and the thickness of the wall of the treatment field required at any time in this field will also be determined and designed from tungsten, lead or iron according to the radiation protection guidelines specified in NCRP Reports 49, 51, 116 and 151 (NCRP, 2014).

Access to the Zap-X radiation shielding system is restricted so that any user is more than one meter from the system circumference without the occurrence of radiation outages. Based on initial design assumptions, radiation levels are measured at a wide range of locations within one meter of the perimeter of the Zap-X system for all major beam locations. Immediate exposure rates, as well as integrated time exposure measurements throughout the treatment period, are determined for 14 equidistant stations around the circumference of accessible areas in Zap-X at a height of 1.2 meters above ground level using the recently calibrated Victoreen model 451 fluke survey Biomedical, Everett, Washington, USA). Measurement sites are closely related to system engineering, as Monte Carlo-based shielding is designed to provide identical radiation levels along a 1-meter circumference line (Weidlich G A., 2017).

A treatment plan is created on the Zap-X TPS radiation shielding system as a cumulative exposure function and delivered using a $20 \mathrm{~Gy}$ reference treatment. Cumulative exposure is measured in the same 14 measurement stations around the system perimeter, as is the case for measuring the exposure rate. An additional measurement site is placed on top of the system at a height of $1 \mathrm{~m}$ above the surface of the treatment field (Station No. 15) (Adler JR, 2017).

The requirements for power, air conditioning and ports monitoring and emergency system must be considered. The design of radiotherapy facilities in consultation with the equipment manufacturer requires (Lechuga, 2017):

- Examination rooms

- Simulation room

- Treatment planning room

- Mold room

- Treatment room

- Waiting areas (Benedict \& et al., 2014) 


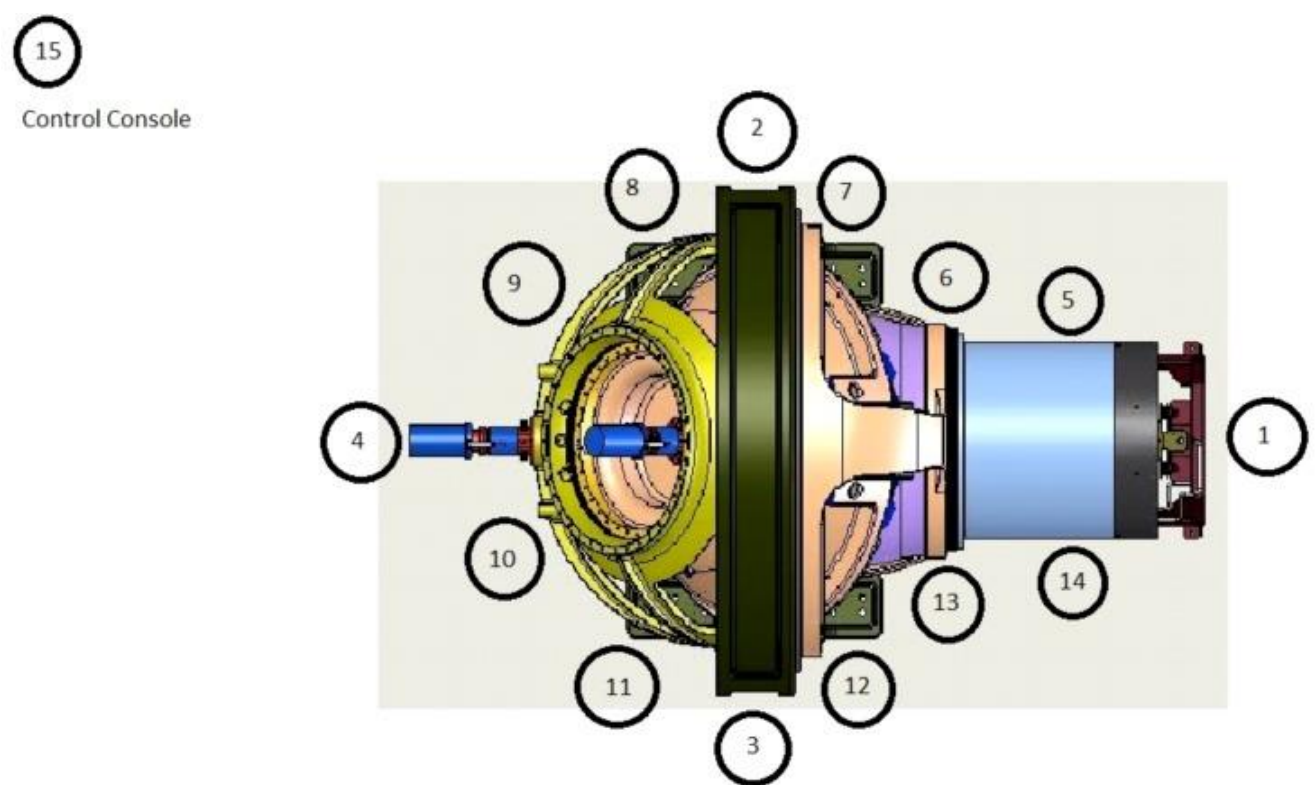

Figure 4: Summary of measurement stations (Weidlich G A., 2017).

\section{RESULTS}

The results indicate that all necessary self-shielding for the audience and neighboring individuals is integrated as part of the system design in order to remove the heavy workload, which eliminates the need to build a dedicated radiation depot. The burden to build a treatment room is removed from the user's organization because Zap-X is equipped with self-shielding protection. The expected radiation levels outside the treatment field are known to meet safety regulations and standards since the shielding requirements for Zap-X have been carefully studied, not dependent on a local designer and have not been improved. The results also indicated that the total time required to prepare the facility is reduced by about six to 12 months during the clinical implementation due to the lack of a need to build treatment warehouses, so the total construction costs will be greatly reduced. And if the shielding buffer for treatment depots is designed to protect radiation workers who are allowed to obtain the annual maximum exceptionally high workloads can be accommodated by adding simple protection provisions, increasing the size of the restricted area, or restricting public access in the immediate vicinity.

\section{CONCLUSIONS}

The radiosurgery system can be protected, resulting in radiation exposure levels considered safe for the public by NCRP standards while operating under a full clinical workload. It has been proven that Zap-X is a self-protecting radiosurgery system without the need for a radiotherapy cache at most user clinical locations

This system is currently under development. Results indicate that a larger case database should be analyzed using an automated version of the proposed methods in order to obtain more detailed design recommendations for a self-protected radiosurgery treatment planning system. After this, a full evaluation of this treatment planning system (once implemented) can be made in the future.

The overall economic benefit of improving access to radiotherapy resources provides a great incentive to invest in areas that are severely short of treatment services. These investments could save 26.9 million years of age for patients treated in these areas. To accomplish this great task, it must be done through a collaborative effort and global partnerships to 
overcome most obstacles. Innovation in self-shielding systems technology for radiotherapy, staff training, insufficient infrastructure improvement, and improved cultural awareness of cancer and radiotherapy will require the effort of many global committees such as the International Atomic Energy Agency, the International Cancer Experts Group, and the International Cancer Union (UICC).

The World Federation and its agreement to have collective action will accelerate great efforts to bridge the gap in reaching the many countries suffering from a lack of radiotherapy services by reducing costs. This option reduces the required level of expertise required for training and personnel in relation to the most recent equipment on the market. These systems will still need a cellar for shielding purposes because it is still a viable option. Therefore, through the evidence mentioned in the research, it can be confirmed that the SS-HQIGRT device is a good option to overcome the ever-present radiation therapy obstacles. The implementation of this system will lead to the provision of a new and cheap device in the market, while reducing the costs of infrastructure and construction at the same time, and providing treatment for the majority of cancer indicators.

\section{REFERENCES}

1. International Atomic Energy Agency. (2017). Availability of radiation therapy.http://www.iaea.org/newscenter/focus/cancercare/dirac2012.pdf (accessed Feb 28, 2017).

2. Adler JR, S. A. (2017). Treatment planning for self-shielded radiosurgery. Cureus.

3. 100I. U. Abhulimen \& J. I. Achebo, "The Use of Adaptive Neuro Fuzzy Inference System (ANFIS) in Modeling the Weld Output of a TIG Welded Pipe Joint", IMPACT: International Journal of Research in Engineering \& Technology (IMPACT: IJRET), Vol. 2, Issue 9, pp. 99-110

4. Atun, R. e. (2015). "Expanding global access to radiotherapy." The Lancet Oncology : 1153-1186.

5. Benedict, \& et al., S. H. (2014). Stereotactic Radiosurgery and Stereotactic BodyRadiation Therapy. CRC Press. Print.

6. Benedict, S. H. (2014). Stereotactic Radiosurgery and Stereotactic BodyRadiation Therapy. CRC Press. Print.

7. Coleman, C., Norman, \& Minsky., \&. B. (2015). "The verdict is in: the time for effective solutions to the global cancer burden is now." The Lancet. Oncology 16.10 (2015): 1146.

8. Sunil Yadav \& Gajanand Sharma, "Improvisation of Data Mining Techniques in Cancer Site among Various Patients Using Market Basket Analysis Algorithm “, BEST: International Journal of Management, Information Technology and Engineering (BEST: IJMITE), Vol. 3, Issue 10, pp. 137-144

9. Elekta. (2017). "3D Image Guided Radiation Therapy Increasingly Found In Community Cancer Centers." PR Newswire: News Distribution, Targeting and Monitoring. PRNewswire, I Nov. 2010. Web. 13 Mar. 2017.

10. Food And Agriculture Organization Of The United Nations,. (1996). International Atomic Energy Agency, International Labour Organisation, Oecd Nuclear Energy Agency, Pan American Health Organization, World Health Organization, International Basic Safety Standards For Protection Against Ionizing Radiation And For The Safe.

11. International Atomic Energy Agency Vienna. (2008). Setting Up A Radiotherapy Programme: Clinical, Medical Physics, Radiation Protection And Safety Aspects.

12. Khan, F. M. (2003). The Physics of Radiation Therapy. Philadelphia: Lippincott Williams \& Wilkins. Print.

13. Lechuga, L. (2017). Simple Xrt: A Self-Shielded Image Guided Radiation Therapy Device. A thesis submitted in partial fulfillment of the requirements for the degree of Master of Science in Physics in the College of Science and Mathematics 
California State Univer.

14. Nazanin Soudbakhsh Moghadam, Anil Kumar H.V \& Muralidhar.S.Talkad, "Therapeutic Potential of Methanolic Extract of Evolvulus Alsinoide Plant for Anti Cancer Activities in Vitro", International Journal of Applied and Natural Sciences (IJANS), Vol. 6, Issue 3,pp. 1-8

15. NCRP. (2014). National Council on Radiation Protection and Measurement - NCRP reports (\#49, 51, 116, 151) [Nov;2017 ];http://www.ncrppublications.org/reports 2014.

16. Rogers DW, F. B. (1995 ). BEAM: a Monte Carlo code to simulate radiotherapy treatment units. [Nov;2017 ];22:503-524.

17. Shultis, J., \& Faw, R. (2000). Radiation Shielding and Radiological Protection Department of Mechanical and Nuclear Engineering, Kansas State University, Manhattan, KS,USA jks@ksu.edufawre@triad.rr.com.

18. Siebers JV, K. P. (2005). The Modern Technology of Radiation Oncology: A Compendium for Medical Physicists and Radiation Oncologists. Vol. 2. Madison, WI: Medical Physics Publishing. Monte Carlo dose calculation for external beam radiotherapy; p. 514.

19. Stewart BW, W. C. (2015). World cancer report 2014. Lyon: International Agency for Research on Cancer, 2014 (accessed Aug $28,2015)$.

20. Van Dye, J. J. (2013). "Accuracy and uncertainty considerations in modern radiation oncology." The Modern Technology of Radiation Oncology 3 (): 361-412.

21. ViewRay. (2015). The MRIdian Advantage. ViewRay. Print.

22. Patrick R Matowa, Felistus Chando, Charles F.B Nhachi \& Mazuru Gundidza, "Evaluation of Anticancer Activity of Steganotaenia Araliacea (Carrot Tree) Barkextract in Cancer Induced Mammary Glands of Female Sprague Dawley Rats”, BEST: International Journal of Humanities, Arts, Medicine and Sciences (BEST: IJHAMS), Vol. 7, Issue 1, pp. 7-16

23. ViewRay. (2017). "ViewRay Receives FDA 510(k) Clearance for MRI-Guided Radiation Therapy System." ViewRay Receives FDA 510(k) Clearance for MRI-Guided Radiation Therapy System. View Ray, 30 May 2012. Web. 13 Ma.

24. Weidlich G A., S. M. (2017). Self-Shielding Analysis of the Zap-X System.9(12). 